\title{
Introduction
}

\author{
Maria Łuszczyńska
}

In recent decades, the process of extending life and improving its quality has become a reference point for a growing group of researchers who define themselves as gerontologists, as well as for an emerging field of gerontology that draws on perspectives from medical science, the humanities and the social, technical and natural sciences.

The ageing of the global population is a fact confirmed by quantitative and qualitative data from the many spheres that are affected by the extension of human life. Here I have in mind such diverse fields of human engagement as (for example) healthcare, new media, the labor market, education, legal systems, social participation, marketing, tourism, the production of goods and social assistance, not to mention pharmacy, automobile travel, air travel, spirituality and culture. Representatives of these and many other spheres in which the presence of older people has a considerable influence are vitally interested in studying the role, impact, purchasing power, tastes, opinions, preferences, needs, expectations and significance of older people as it relates to their presence and engagement. It can thus be generally asserted that all those areas of activity and knowledge in which older people play a meaningful role have a gerontological character, despite the fact that gerontology has so far reserved for itself a narrower field of research that is connected to the ageing processes of living beings - and in particular, of the human being (Zych, 2010).

For the purposes of this publication, such a narrow understanding of gerontology is reversed, and (in accordance with N.R. Hooyman's view), "gerontologists" are taken to include researchers and practitioners in the fields of biology, nursing, medicine, criminology, dentistry, social work, physical and occupational therapy, psychology, psychiatry, sociology, economics, political science, architecture, geography, pharmacy, public health, housing and anthropology (Hooyman and Kiyak, 2011). This list could also be extended to include pedagogues and educators, caregivers and assistants, social animators, social policymakers, specialists in marketing, media and fashion and other persons whose professional and social activities are centered around older people. This means that gerontology may be the most multidisciplinary (as well as one of the youngest) fields of scientific knowledge. ${ }^{1}$ This publication is addressed to all such people who are engaged with this field.

The dynamic process of global ageing brings many new questions, problems and challenges whose answers are sought in the course of research. Such issues absorb those who (more or less) consciously participate in research activities on a large or small scale. The question of what is really being studied when research deals with ageing, old age and the elderly - as well as the question of why and how such studies are conducted - are fundamental questions related to the methodology of gerontological research, regardless of the specific field involved. Meta-reflection on gerontological research must engage in the same discussion that continues to reverberate through mainstream philosophy of science. As in 
that case, the starting point may be a desire and search for objectivity in scientific research. However, it readily becomes apparent that the postulate of objective knowledge was already being overturned by Kant and his epistemological Copernican revolution. Since his time, philosophers and scientists have continually discussed the subject of science. The result of these debates is a general compromise known as the "scientific method" (Popper, 1959: 28), which involves the development of methods for collecting relevant data, creating logical generalizations that would explain such data and, finally, formulating ways to test those generalizations. The scientific method makes it possible to obtain knowledge that is "scientific" - i.e., proper, true and consistent with reality. In the opinion of twentieth-century philosophers of science (like, for example, T. Kuhn and I. Lakatos), it is only by observing the rules of this method that one can gain real knowledge about the world. The scientific method is considered to be a highly specialized tool for describing, explaining and understanding the world, which scientists employ in seeking answers to the questions posed and building a non-arbitrary, reliable and coherent image of the world. Traditionally, it has been said that it is a way of seeking the truth (Bronk, 2006); social, subjective, psychological and cultural elements obscure such truth and lead to a decrease in the quality of scientific knowledge. It might be said that the scientific method was the final weapon developed as a means of trying to answer the question of whether there is a specifically "scientific" approach to understanding the world. Here I do not want to delve into the history of the development of the scientific method or discuss the detailed debates about it conducted by philosophers of science (see Grant, 2005; Russo, 2005). However, it can be noted that when we presume the scientific method - with its strictly formalized procedures, advanced formal tools and cult of quantitative methods - to be the only means for learning the truth that might be acceptable to all scientists, this leads to a depreciation of qualitative methods, the humanities and the social sciences. It was thus only a matter of time before those fields would attempt to introduce other scientific paradigms. The trends of analytical philosophy (T. Kuhn, P. Feyerabend) and phenomenological philosophy (E. Husserl, M. Heidegger, H.-G. Gadamer) contributed to the recognition that the rejection of existing theories and adoption of new theories is determined largely by social factors and various research paradigms. Philosophers emphasize the social nature of scientific communities (T. Kuhn) and the "myth of method" (P. Feyerabend), which recognizes that the development of science thus far has only been possible because of approaches that contravened the sanctioned views of the scientific method. In the latter philosopher's view, the only rule that should be defended in science is the "anything goes" thesis which postulates that all ways of researching - even those that are "crazy" - are equally justified (Feyerabend, 1975). Similarly, Gadamer draws attention to the fact that the methodical way of knowing (as it is understood in science, and particularly in the natural sciences) cannot be applied in every field: "valuable" ways of knowing cannot simply be identified with "methodical ways". The scientific method only is not the only way to the truth (as various "scientisms" have claimed); rather, it sometimes even inhibits the development of knowledge (Gadamer, 1993).

And so, spurred by the recognition that other perspectives - whether cultural, social, individual, intersubjective, temporal, spatial or otherwise - possess the same level of importance (despite the lesser verifiability of their results), there have emerged over time other research approaches like the cognitive, existentialist, feminist, constructivist, phenomenological, postmodernist and deconstructivist perspectives. Researchers are willing to adopt a wider range of perspectives and to accept the practices, systems of practical activity and systems of attitudes, behaviors and beliefs that emerge within these perspectives. Along with them, material and cultural products - methodological, political and normative 
perspectives - enter the boundaries of research. The studied reality is not seen to be a finished thing given to human knowledge once and for all time, but as something created and constructed - which means that it also can be (and often is) imagined as something better; it is something that can be improved. Through such rebellion against the scientific method, it is possible to first recognize some separate reality that had been overlooked, and then somehow elaborate methods for studying it, after defining perspectives, practices and products. In this way, methods are shaped not only by that which is social and collective but also by that which is individual; they are formulated by (but also formulate and format) that which is social. The expectation that method will be something finished, structured and proven is - while dear to the creators of the scientific method - far from the minds of such researchers who are penetrating the human world and, within it, phenomena like ageing in a multidimensional perspective. They recognize that the human world is a realm that is boundless, open, multi-threaded and, to some extent, concealed. They agree to discover it piece by piece, in a fragmentary, non-total manner, but also (importantly) with a recognition of what is practical. In this fashion, a great transformation of science - and of that which is recognized as scientific - is underway. The horizons of researchers' interests are no longer defined by total systems of premises, axioms, laws, scientific theories, various conflicting "-isms" and a hope of absolutely determining the correctness of a given theory; such horizons are now being expanded by networks of practice, relationships, multiplicity, diversity, transgressiveness, praxeology, agility, technology, globality, selectivity and depth of study.

Such are the horizons that define our area of inquiry. It is a moment in the development of research and its methodology in which we move beyond extremes like nature/culture, human/inhuman, micro/macro and physical/mental and examine things like relations, dependencies, processes, actors, objects, institutions and strategies, with regard to their universalistic, holistic and transrelational nature.

Our location is another issue. Each of us is located somewhere, in some space, as well as in time and in a systemic, cultural and social context. This does not mean that research from such a perspective will be subjective; however, in the search for objectivity, one needs to be aware of one's "social location", which is precisely an embedding in a certain reality of time, space and culture. The awareness that full objectivity is not achievable that objectivity is always, in some sense, partial - underlines the need not only to recognize one's social location but also to subject social knowledge bases to research. Knowledge creators, researchers and theorists are always part of what they study, and the methods they use should take this element into account, rather than pretending that it doesn't matter (Harding, 1993).

This book has been co-created by almost 50 authors from 11 countries around the world. Their social locations constitute a map with a fairly large geographical area. Among these "locations" - understood as both the terrain where studies have been performed as well as the terrain for scholarly, cultural and social reflection - we should mention Norway, the United States, Turkey, Portugal, Brazil, New Zealand, Canada, Ireland, South Africa, India, Chile, Australia, France, Germany, Sweden, Greece, the United Kingdom, Poland, Italy, the Netherlands, Taiwan, France, Japan, Israel, Austria, the Hong Kong Special Administrative Region of the People's Republic of China, rural China and Belarus. Thanks to such a diverse collection of research areas (and the international diversity of the researchers and methodologists who are the volume's authors), a reader will be able to encounter social locations on a global scale, while also having the opportunity to carry out his or her own meta-reflection on the parallel methodologies employed in various places around the world. 
This can serve as the beginning of a discussion about reconciling various research patterns - but it also demonstrates the extraordinary richness of the fundamental field of human activity that is the conducting of research.

The intention of this publication's authors was not so much to create a textbook from which students in various countries of the world who are studying gerontological questions could draw instructions, definitions, tips and guidelines for conducting their research; rather, the objective has been more about capturing the wealth of methodological thought and encouraging the reader to undertake his or her own theoretical search. Above all, the book aims to share its authors' own experiences and research reflections in order to inspire ever better research-based representations - whether they be objective, intersubjective, subjective, known, constructed or transformed - of the reality of the ageing world. It is hoped that the publication can contribute to discussions on the methods and subjects of studies, applications, collaboration and ways of drawing on the achievements of other research disciplines and fields.

One main purpose of the publication is to explore the diversity of methodological aspects of research around ageing and to contextualize it within a discussion about which of the main methodological paradigms more effectively and reliably illustrate the phenomenon of ageing. This goal fits within the ongoing conversation between researchers employing qualitative and quantitative paradigms. The objective of the publication is not so much an exchange of arguments involving these two approaches, but rather the reconciliation of the conditions of their coherence and complementarity. Interdisciplinary and social perspectives, as well as the qualitative dimension of research on old age, are emphasized.

The editorial decision was made to highlight this wealth of reflection on gerontological research through arrangement of the book's content into particular parts. The first part includes chapters that consider gerontological research in relation to (and using) the methodological output of other fields that study and describe the world, such as philosophy, sociology, legal science, economics, and management and marketing.

The book opens with a chapter from Zbigniew Woźniak that highlights a multidisciplinary perspective in the search for context for research into old age. It shows the correlation between the approaches of gerontology and other scientific disciplines and their tangent points. The author formulates a useful tabular summary about research priorities in gerontology, the functions and aims of gerontological diagnosis, and categorial profiles of older adults.

In the chapter by Angus McMurtry and Jenny Sasser, the authors illustrate the difference between multidisciplinary and interdisciplinary/transdisciplinary approaches to ageing and gerontology. It is a rare phenomenon in gerontological research and reflection to investigate deeper interdisciplinary interdependencies and to attempt to formulate a perspective that considers in depth the interdisciplinary relationships existing in research on old age. However, it seems that gerontological studies constitute a fruitful material for such reflections, due to their "genetic" involvement with many disciplines.

In their chapter, Martine Lagacé, Najat Firzly and Alura Zhang rely on a qualitative methodology for a scoping review. Such scoping reviews allow for the identification of broad and common themes related to a topic. In this particular case - in which self-reported scales of ageism in the workplace were studied - the scoping review has made it possible to assess the psychometric properties of the scales as well as the dimensions of ageism that they address.

Marcel Mérette and Julien Navaux investigate ageing in the population using National Transfer Accounts (NTA), showing how the NTA is an extension of the System of National Accounts (SNA). Such systems make it possible to implement complete and consistent 
techniques for measuring the economic activity of a nation or a region. SNA accounts quantify in a rigorous way fundamental economic variables such as production, consumption, savings and investment. NTA extends SNA at the individual level for each age. Such extension is necessary for a deep investigation of the economic implications of population ageing. About 90 countries have developed NTA at the national level, and the data provided by these countries allows interesting international comparisons. A reference manual published by the United Nations in 2013 describes the technical details of NTA.

Anna Szafranek shows that a methodology for research into the protection of older adults' fundamental rights should include: (1) the legal perspective (research into the implementation and effectiveness of particular legal acts), (2) the administrative perspective (research related to the effectiveness of institutions that reinforce the law) and (3) the perspective of the relationship between institutional and socio-economic factors. All the aforementioned perspectives ought to intertwine, in order to present the phenomenon in the broadest possible way. Research into the protection of the fundamental rights of older adults was carried out using a qualitative, narrative, gerontological approach, and the chapter presents this context.

In her chapter about ethical perspectives in gerontological research, Maria Łuszczyńska argues that every well-constructed methodological framework must also take into account ethical assumptions. The chapter illustrates the way in which these assumption permeate every stage of the research process; it also proposes audit questions for each stage that verify a given research procedure's orientation toward the ethical dimension.

The second part of the book presents various research perspectives. Here the intention was to move beyond the age-old methodological dichotomy of quantitative and qualitative approaches and to show mixed models, along with other perspectives such as the cultural model, life course perspective, longitudinal statistical models and approaches to studying sensitive issues. Among other topics, this part investigates previous considerations on the inclusion of a researcher in methodological reflection, especially when the researcher intends for his or her research activity to encompass a larger segment of reality.

Combining design research and social innovation methodologies, Yanki Lee focuses on the observations of T. Blair and M. Minkler (2009) on why researchers do not conduct Participatory Action Research (PAR) but continue to employ the more traditional investigatordriven gerontological methodology.

Barbara Szatur-Jaworska shows that the life course perspective can be adopted in research belonging to either the normative or interpretative paradigm (according to the division proposed by Thomas P. Wilson). The chapter describes two life course conceptualizations: (1) as a normative social construct and (2) as an empirically experienced process of biological, psychological and social changes in an individual's life.

Annie Robitaille and Graciela Muniz Terrera consider methodology, with a focus on the different statistical models used to study the ageing process when longitudinal data is available. More specifically, the latent growth curve model, multivariate latent growth curve model, latent growth curve model with a time-varying covariate, latent class analysis, growth mixture model, multi-state model and change point model are discussed in the context of ageing.

Piotr Czekanowski deals with concepts generally employed in the methodology of social research, such as quantitative, qualitative and mixed approaches, research methods, techniques and tools. The considerations presented in his chapter touch on the differing treatment of quantitative and qualitative methods, which has been consciously reduced primarily to a technical (i.e., neutral) distinction between these methods. In the background 


\section{M. Łuszczyńska}

of the issues presented in this chapter, the author also references the theoretical positivist approach, usually associated with quantitative methods and focused on the standardization of research, and the humanistic approach associated with various interpretative trends.

Using qualitative methods (in-depth interviews and narrative interviews), Małgorzata Halicka and Jerzy Halicki reflect on research into sensitive topics within the area of violence. They show how to prepare, conduct and summarize such research, whose narrators find themselves in a very specific situation. They also analyze the importance of the research attitude and action when issues of harm are on table. Their study involved research conducted in Poland as well as one project in Belarus.

In her chapter, Carolin Kollewe discusses how research on old age can be innovated through perspectives that have become paramount in the social and cultural sciences in recent years, in particular those used in material culture studies and science and technology studies. In concrete terms, this calls for a qualitative, in-depth, reconstructive investigation. Regarding methodology, such an approach starts from the various material objects that are employed in people's everyday social practices and looks at related representations. On the one hand, such an approach analyzes the diverse social meanings that are both attached to objects by the individuals using them and materialized in these objects. On the other hand, by investigating social practices in which these objects take part, it is possible to unravel how people and things co-construct old age and ageing practically. To analyze how age and ageing are constituted, the study that Kollewe presents as an example employs the methods of object description, participant observation and interviews. Concomitantly, her analysis is based on an inductive and hermeneutical approach, partly influenced by the methodology of grounded theory (Glaser and Strauss, 1967) and its latter elaboration as situational analysis (Clarke, 2005).

Henglien Lisa Chen has prepared a chapter that fills a significant gap in the discussion of qualitative research methods and methodologies in cross-national comparisons by a solo researcher. It details the challenges and opportunities inherent in conducting cross-national research in long-term care of older people by a solo researcher using qualitative methods, which has been rarely been carried out or discussed. The chapter identifies and addresses the central challenges of time and space, comparability and the culture and language encountered in the research process, as a means of illustrating this distinctive methodological approach. The chapter draws on a cross-national study from a $\mathrm{PhD}$ project.

The third part of the book deals with issues connected with the use of research results. The authors consider the role of studies aimed at improving not only living conditions, health, physical activity and fitness but also mental condition and social relations. These chapters contribute to a discussion of the role that a study's utility should play in research, while also illustrating various tools like the SoBeezy program gerontopoles and social marketing, which can constitute a meta-reflection of the scope, meaning, purpose and consequences of the treatment of ageing research as application research.

The team of French colleagues comprising Linda Cambon, François Alla and Karine Pérès considers ageing interventions as complex interventions. Hence, they refer to the paradigm for the evaluation of complex interventions developed in the guidelines of the Medical Research Council (MRC). In particular, this highlights the process of evaluation that is necessary for understanding how complex interventions work. This evaluation helps define the conditions of transferability of interventions by understanding their mechanisms of effect. More precisely, the authors argue that one of the various existing evaluation paradigms - that of theory-driven evaluation - makes it possible to understand these mechanisms and should be thus integrated in all evaluation designs. Moreover, they recognize the 
social utility of intervention research and advocate for the consideration of real-world conditions in intervention design and evaluation through viability studies.

Elise Verot and Alexandra Lelia Dima demonstrate how mixed-methods designs are increasingly being applied in order to combine different types of complementary evidence for developing new care protocols and tools. Implementation science is emerging as a rich set of practical tools for generating evidence that can guide organizational change processes at different levels of the healthcare system, and the authors discuss the research procedures that support it.

Though there is now agreement that physical activity (PA) is effective in practice, there are many barriers to participation. Indeed, several such barriers exist among the elderly, such as a belief that one is no longer able to participate because of a loss of physical capacity, an image of sport as being for young, healthy people, and poor awareness of the tailored activities on offer. Luc Goethals, Nathalie Barth, David Hupin, Frederic Roche, Karine Gallopel-Morvan and Bienvenu Bongue argue that campaigns promoting physical activity generally have the ultimate goal of changing the behavior of individuals. However, between campaign exposure and behavioral change, several steps are needed - i.e., changing beliefs, attitudes and intentions. Very often, evaluation of the effectiveness of preventive campaigns is limited to measures of recall of the message and of appreciation of advertising. But is knowing the key recommendations that promote physical activity among seniors enough to change behaviors? Social marketing research emphasizes the need to focus on behavioral change to identify effective levers for preventive action.

The next chapter presents the gerontopoles as a unifying structure of all the actors of a region. In this context, collaboration is essential for carrying out projects. Theoretical approaches related to such collaboration have made it possible to define different concepts such as transactive memory, coordination, collaboration and cooperation. The research by Nathalie Barth, Clara Pizzolo, Solène Dorier, Régis Gonthier and Thomas Célarier demonstrates the effectiveness of collaborative work within teams and the partnership work that appears essential to the success of gerontopoles. Finally, the methodology of the Living Lab seems to be central in the actions of gerontopoles that wish to place the user at the heart of projects. Specific research on the different Living Labs of gerontopoles could advance the work of this first chapter to investigate the importance of this innovative methodology.

Daniela Wetzelhütter, Katrin Hasengruber, Renate Kränzl-Nagl and Tina Ortner develop a model based on the modified stress-strain concept (Metz and Rothe, 2017) to measure the influences upon and consequences of subjectively perceived work-based stressors among people working in nursing and care. Their aim was to compare different nursing and care concepts to identify differences in people's subjective perception of work-related stressors. A summative evaluation design was therefore applied: more precisely, a semiexperimental design with a control group and an experimental group, collecting data in two waves ("before and after" measurements). This permits the simultaneous evaluation of the experimental group (in which a new concept is introduced) in one or more care homes and the control group (in which the specific concept is not implemented) in comparable care homes. Changes over time can thus be studied under controlled conditions.

Einav Segev and Yael Hochman, who are qualitative researchers, have grounded their research methodology in postmodernist and constructivist theories of reality and of how it should be explored. The model they use in the chapter has thus enabled them to observe participants' subjective perception of their loss and of family relationships. This was accomplished within the phenomenological tradition that seeks to explore phenomena from 
the perspective of those who experience them. The model is based also on the ecosystems theory (Bronfenbrenner, 1979) that enables exploration of relationships in various environments - here, the subjective perception of participants in the research, dyadic relationships and integrative observation of all the families that participated in the study.

The fourth and final part of the book focuses on our primary sources of knowledge about ageing - i.e., on the persons directly affected by this process. This part begins with a chapter about involving older people themselves in research into old age, with the authors pointing out the strengths and weaknesses of this research technique. In this part of the book, one can also learn how to conduct research with residents of nursing homes who have impaired communication or are less educated and learn about the Photovoice technique as used in participatory research.

The chapter prepared by the Hong Kong research team comprising Ke Chen, Justin Chun Ting Cheung, Joy Juan Wang and Vivian Wei Qun Lou is a part of a growing body of studies that involve older adults as co-researchers in the study of ageing - a participatory peer research method. As described by Glanz and Neikrug (1997), "Those who are intended to benefit from the research should be involved from its inception in the formulation of the research agenda and conceptualization of the research questions and design, as well as its implementation." Critical questions are immediately raised: in what kind of research activities and to what extent should older people be involved? Who should shape the research agenda and carry out the research? What are the roles of older people and academic researchers? What is the impact on the research process and validity of involving lay older people as research partners? How should the ethical and practical issues and risks of using this method be managed? There are various strands to the discussion, and many gerontologists are hesitant to embrace this method, because a coherent and systematic framework has not yet been developed, and there are very few models of good practice on which to draw.

In their chapter, Linda J. Garcia and Louise Bélanger-Hardy focus on participant selection in research, and more specifically on the involvement of older adults with difficulties in communication. Until recently, actors within the research community have been hesitant to include participants whom they perceive as lacking the capacity for providing informed consent because of age or functional limitations or to adapt their protocols to those who may struggle to offer information during language-dependent tasks. According to the authors, this trend is changing, and researchers, institutional review boards and granting agencies are increasingly encouraged to be more inclusive in their practices. Relying on an ethico-legal framework, Garcia and Belanger-Hardy consider some of the perceived limits on participation, including capacity, informed consent, substitute decision-making and, finally, the notion of vulnerability. After noting that international human rights instruments provide a strong basis for the inclusion of older adults in research protocols, they argue that researchers must provide a "safe space" for participants to fully take part in research projects. Drawing on the developing literature on the inclusion of dementia patients in research and the extensive literature on aphasia, the authors highlight a number of suggested strategies to increase participation in research of older adults with communication challenges while ensuring their safety and well-being.

In exploring the life experience of rural older adults, Shirly HZ Chen and Vivian Wei Qun Lou use a life-story interview strategy that was facilitated by the life story method and oral history method, with technical matters having been adjusted. Unlike a traditional lifestory approach or oral history method that emphasizes the "natural emergence" of participants' life experience, this strategy suggests a starting point, a triggering "linguistic frame" and an "acceptable interview field" to promote interview involvement. 
Although a growing number of studies deal with quality of life among nursing home residents, only a small number examine the practical aspects of such research in any detail. Against this background, Renate Kränzl-Nagl and Daniela Wetzelhütter focus in their chapter on the preparation and implementation of research activities in nursing homes for the elderly, based on experiences of research carried out in Austrian nursing homes. Special attention is given to methodological challenges with regard to the individual steps in the process of investigation. This chapter's contribution is also characterized by a multiperspective approach that considers numerous actors in the context of research in nursing homes for the elderly. The model of factors influencing empirical research with nursing home residents developed by Ackermann (2005) provides the theoretical approach for the analysis undertaken in this chapter.

Photovoice, a participatory research methodology, facilitates the elucidation of the experience of certain populations and groups through the use of photography. Building on general principles for how to use this methodology, this study conducted by Frances Lu Yang, Vivian Wei Qun Lou and Carman Ka-Man To has flexibly adapted it for investigating the specific population group of male caregivers in Hong Kong. This adaptation affects how Photovoice is defined in the context of this study, in that the collaboration between researchers and participants and the empowerment of participants should be emphasized. Rooted in the theoretical assumptions of critical consciousness, feminist theory and phenomenology, Photovoice is redefined in this study.

The division into parts adopted for the book - along with the content of the individual chapters - tries to capture those diverse aspects of gerontological research that may develop over time as subdisciplines of gerontology. It is also worth noting what has been left out of the book: nowhere will the reader encounter a tabular summary of old age research methods. Such a comparison could be made with regard to the thought of the authors of this book, but we recognize that it would be only a fragment of the possible research reality. Moreover, our aim was to prepare a publication that is primarily inspirational rather than educational in nature; we have therefore consciously avoided a framework that primarily organizes reflections from gerontological research on the basis of their use of, for example, feminist, historical, existential, naturalistic and other perspectives - although elements of these perspectives appear in individual chapters.

How, then, should "methods" and "methodologies" be understood when introducing a book whose title speaks of methodological challenges? Most generally, method can be understood in accordance with its etymology as following someone, as moving along a path (from the Greek methodos, or meta and hodos). Understood broadly, it means a way or path of conduct, which is divided into steps or stages that must be performed in a certain order so as to achieve a certain goal, to accomplish a given task. Scientifically understood, method indicates a way of solving theoretical or practical problems (Kotarbiński, 1961: 516). Functionally, a method is a system of more or less ordered activities (i.e., occurring in a specific sequence) that serves to increase the efficiency and economy of a certain action. When it comes to a directive or regulatory understanding, method is a set of more or less uniform provisions (i.e., rules, directives, guidelines or maxims) that determine the course of a certain action, facilitating the more effective and economical implementation of a specific goal or solution for a specific task. The term "method" (methodus) appeared in Latin culture in the twelfth century as a translation or calque of the Greek methodos, gradually displacing (in knowledge-related contexts) earlier translations that employed terms like via, ars, ratio, disciplina, doctrina, regula, compendium, habitus, scientia and techne. In the concept of method there is something of a norm, but also of art and invention (Bronk, 
2006). Nowadays, method is associated with a mode of action, the achievement of goals, a technique of operation, a technology of operation or instructions for operation. What is important is that method is related to action; it has a practical dimension and therefore can be modeled, modified and transformed. It is therefore creative; that is its general context.

What sort of understanding of methodology do the authors of this publication present? We define methodology as a framework and justification for pursuing research. Methodology must suit the phenomenon that one is studying and the research context, including one's own position as a researcher. We would also argue that interdisciplinary integration and transdisciplinary collaborative action constitute valuable methodological frameworks. Methodology places value on conducting research in a particular way and determines how studies are conducted. Moreover, the authors emphasize the practical dimension of research. It should serve some goal by contributing to positive change for a specific population along a particular social, cultural, political, economic, health or other dimension. This reality brings with it an emphasis on reliability in preparing the whole process, as well as an awareness of fact that choosing the purpose for which the research effort will be undertaken and (later) choosing the research methods and sources of knowledge constitute the most "authorial" moments of the research process for one studying old age, in which researchers can explore the limits of their imagination.

It is undeniable that the researcher permeates the entire research process. Such a researcher moves in a world with a great diversity and abundance of research fields, topics, methods, tools and innovative approaches, as well as of cultural circles and social locations. The quality of the entire research process depends on the researcher's inventiveness, insightfulness, dependability and courage, as well as on the ability to cooperate in an investigating team. The art of research (including gerontological research) is dependent on the manner in which a researcher deals with diversity and differences - between the researcher and the source of knowledge, between his or her attitude and reality, between the researcher and other people's ideas - and on the way in which such diversity and differences are found. A researcher is supposed to transform the world, expanding borders (if only to a small degree) and creating alternative and better solutions. This does not mean that one must accept that everything is possible and that the world consists of infinite possibilities and that one just needs to find a method that is innovative enough in order to turn it upside down. And yet, a wide range of research methods is at the disposal of the researcher, and it is worth studying the experiences and reflections of other practitioners of research on old age in order to search for and find inspiration.

This publication is international in character and constitutes part of the project known as the "Global Ageing Research Partnership". The project is funded by the Polish National Agency for Academic Exchange (NAWA) within the International Academic Partnerships program. The project partners are: the Pontifical University of John Paul II in Kraków (Poland), the project leader; the University of Applied Sciences Upper Austria (Austria); Jean Monnet University (France); the LIFE Research Institute, University of Ottawa (Canada); and the Sau Po Centre on Ageing, Hong Kong University (Hong Kong).

Finally, on behalf of all the individual chapters' authors, I would like to thank our publisher, Taylor \& Francis, for their openness to our idea for the book and for the trust that they showed throughout the entire process of creating and publishing this volume. I would particularly like to thank Neil Jordan, Senior Editor with Routledge, for demonstrating such care for the quality of this publication at every stage of its development. I would also like to thank the many others at the publishing house who have worked on this book. On behalf of each of the authors, I express our gratitude to all those important people in each 
of our lives who, by providing daily support, mobilize us for work and for boundless exploration.

It is my hope that all readers will find in this publication a source of inspiration and encouragement for working to change the situation of older persons, because the changes that we are able to make now will lay the groundwork for a better experience of ageing for future generations - and for ourselves.

\section{Note}

1 The term itself was created in 1903 by Élie Metchnikoff (1845-1916), a Russian microbiologist living and working in France, for whom "gerontology" was understood as the science of old age.

\section{References}

Ackermann, A., (2005). Empirische Untersuchungen in der stationären Altenhilfe: Relevanz und methodische Besonderheiten der gerontologischen Interventionsforschung mit Pflegeheimbewohnern. Münster: LIT.

Blair, T. and Minkler, M., (2009). Participatory action research with older adults: key principles in practice. Gerontologist, 49(5), 651-662. doi:10.1093/gerant/gnp049.

Bronfenbrenner, U. (1979). The ecology of human development: experiments by nature and design. Cambridge, MA: Harvard University Press.

Bronk, A. (2006). Metoda naukowa. Nauka, 1(2006), 47-64.

Clarke, A.E. (2005). Situational analysis: grounded theory after the postmodern turn. Thousand Oaks, CA: Sage Publications, Inc.

Feyerabend, P. (1975). Against method. New York: New Left Books

Gadamer, H.-G. (1993). Prawda i metoda. Kraków: Inter esse.

Glanz, D. and Neikrug, S. (1997). Seniors as researchers in the study of ageing: learning and doing. Gerontologist, 37(6), 823-826. doi:10.1093/geront/37.6.823.

Glaser, B. and Strauss, A. (1967). The discovery of grounded theory. Chicago, IL: Aldine.

Grant, E. (2005). Średniowieczne podstawy nauki nowożytnej, w kontekście religijnym, instytucjonalnym oraz intelektualnym. Warszawa: Wydawnictwo Prószyński i Spółka.

Harding, S. (1993). Rethinking standpoint epistemology: what is "strong objectivity"? In: L. Alcoff and E. Potter (eds), Feminist epistemologies. New York: Routledge.

Hooyman, N.R. and Kiyak, H.A. (2011). Social gerontology: a multidisciplinary perspective. 9th ed. Boston, MA: Pearson Education.

Kotarbiński, T. (1961). O pojęciu metody. In: T. Kotarbiński, Elementy teorii poznania, logiki formalnej i metodologii nauk. Wrocław, Warszawa, Kraków: Ossolineum.

Metz, A.M. and Rothe, H.J. (2017). Screening psychischer Arbeitsbelastung: Ein Verfahren zur Gefährdungsbeurteilung. Wiesbaden: Springer Fachmedien. Available at: https://link.springer. com/content/pdf/10.1007\%2F978-3-658-12572-1.pdf [Accessed 19 January 2018].

Popper, L. (1959). The logic of scientific discovery. London and New York: Routledge.

Russo, L. (2005). Zapomniana rewolucja: grecka myśl naukowa a nauka nowożytna. Kraków: Universitas.

United Nations (2013). National Transfer Accounts manual: measuring and analysing the generational economy. Department of Economic and Social Affairs, Population Division, United Nations, New York.

Zych, A. (2010). Leksykon gerontologii. Kraków: Wydawnictwo Impuls. 